\title{
Identification of novel factors that increase enzymatic saccharification efficiency in Arabidopsis wood cells
}

\author{
Misato Ohtani ${ }^{1,2, *, \dagger}$, Vasagi Ramachandran ${ }^{1, \dagger}$, Takuya Tokumoto ${ }^{1}$, \\ Arika Takebayashi' ${ }^{2}$, Ayumi Ihara ${ }^{2}$, Tomoko Matsumoto ${ }^{2}$, \\ Ryoko Hiroyama ${ }^{2}$, Nobuyuki Nishikubo ${ }^{2}$, Taku Demura ${ }^{1,2, * *}$ \\ ${ }^{1}$ Graduate School of Biological Sciences, Nara Institute of Science and Technology, Ikoma, Nara 630-0192, Japan; \\ ${ }^{2}$ RIKEN Center for Sustainable Resource Science, Yokohama, Kanagawa 230-0045, Japan \\ *E-mail: misato@bs.naist.jp Tel: +81-743-72-5460 Fax: +81-743-72-5469 \\ **E-mail: demura@bs.naist.jp Tel: +81-743-72-5460 Fax: +81-743-72-5469
}

Received October 7, 2017; accepted November 7, 2017 (Edited by M. Yamaguchi)

\begin{abstract}
Developing methods to efficiently convert lignocellulosic polymers, i.e. cellulose, hemicellulose, and lignin into user-friendly carbon resources, such as fermentable sugars, is critical for improving plant biomass utilization. Here, we report the identification of genes that increase enzymatic saccharification efficiency in cultured Arabidopsis wood cells. We overexpressed a set of genes that were upregulated during the early stages of in vitro xylem vessel cell differentiation, including transcription factor and CAZYme genes, in Arabidopsis and tested their effects on enzymatic saccharification efficiency. Of the 96 transgenic seedlings sampled, 37 and 17 lines showed significant increases and decreases in glucose yields, respectively. Further analysis of 20 overexpression lines with high glucose yields in seedling samples indicated that compared to wild type, the glucose and xylose yields from inflorescence stem samples were higher in lines overexpressing genes encoding BETA-XYLOSIDASE 2, UDP-GLUCOSYL TRANSFERASE 88A1, AT3G15350 (a class GT14 glycosyltransferase protein), and the Dof-type transcription factor Dof4.6, whose detailed molecular functions have not yet been characterized. No apparent defect in growth or inflorescence stem structure was detected in these overexpression lines. Therefore, these four genes might represent novel factors that can be used to increase saccharification efficiency in wood tissues without negatively affecting total biomass production. Furthermore, our results confirm the validity of our screening strategy for isolating factors related to the saccharification efficiency of lignocellulosic biomass.
\end{abstract}

Key words: CAZyme, lignocellulosic biomass, overexpression, saccharification, transcription factor.

Lignocellulosic biomass is composed of cell wall polymers, such as cellulose and hemicellulosic polysaccharides, and the phenolic polymer lignin. These renewable biopolymers are considered to represent potential sustainable bioresources. Improving the industrial utility of these polymers would greatly facilitate sustainable development efforts (Isikgora and Becer 2015; McCann and Carpita 2008; Pauly and Keegstra 2010). One of the difficulties in utilizing lignocellulosic polymers is the complicated secondary cell wall (SCW) structure of woody cells, which greatly affects the processing of lignocellulosic biomass into fermentable sugars, i.e., saccharification (Isikgora and Becer 2015; Khare et al. 2015). Many studies have aimed to isolate novel genetic factors with positive or negative effects on SCW properties; one method involved screening libraries from transgenic or mutant Arabidopsis thaliana (Arabidopsis) plants for novel factors that enhance enzymatic saccharification efficiency (Hisano et al. 2009; Loqué et al. 2015; Sakamoto and Mitsuda 2015; Van Acker et al. 2013). In the current study, in an attempt to isolate additional novel factors that alter SCW properties, we performed overexpression analysis of woody cell differentiation-related genes in Arabidopsis.

Xylem vessel cells, a constituent of the waterconducting xylem vessel tissue, are a type of woody cell (Myburg et al. 2013). Based on the available transcriptome data of Arabidopsis in vitro system (Kubo et al. 2005), we selected genes that are upregulated during xylem vessel cell differentiation, which are expected to be involved in SCW biosynthesis (Supplementary Figure S1; Supplementary Table S1). Overexpression FOXhunting plasmids harboring full-length cDNA clones corresponding to these genes, which were developed

Abbreviations: CAZyme, carbohydrate active enzyme; FOX line, full-length cDNA overexpressing line; GH, glycoside hydrolase; GT, glycosyltransferase; SCW, secondary cell wall; TF, transcription factor.

${ }^{\dagger}$ These authors contributed equally to this work.

This article can be found at http://www.jspcmb.jp/

Published online December 21, 2017 
by the Plant Genome Project of RIKEN Genomic Sciences Center (Seki et al. 1998, 2002), were obtained from RIKEN Bioresource Center. In these plasmids, the cDNA sequences of interest are expressed under the control of the CaMV $35 \mathrm{~S}$ promoter for overexpression in Arabidopsis plants (Ichikawa et al. 2006). We introduced these 96 constructs into Arabidopsis plants by the floral dip method (Clough and Bent 1998) and obtained more than 10 independent $T_{1}$ plants per construct. Based on the expression levels of the induced genes, we selected three independent lines as overexpressors, which we subjected to further analysis.

We performed enzymatic saccharification analysis using 14-day-old seedlings of both the overexpressor and control lines expressing the intron-containing YFP reporter under the control of the $35 \mathrm{~S}$ promoter (Numata et al. 2014; Ohtani et al. 2013). We selected 20 $\mathrm{T}_{2}$ 14-day-old seedlings based on hygromycin resistance, froze them in liquid nitrogen, and subjected them to enzymatic saccharification using a mixture of cellulase from Trichoderma reesei ATCC 26921 (SIGAM, C2730) and cellobiase from Aspergillus niger (SIGMA, C6105) according to the methods of Okubo-Kurihara et al. (2016). The glucose yields in the transgenic lines were $76-160 \%$ that of the control plants (Supplementary Table S1), and 37 and 17 genes in the overexpressors had significant positive and negative effects on enzymatic saccharification efficiency, respectively (Table 1; Supplementary Table S1). The overexpression of half of the genes examined significantly altered the enzymatic saccharification efficiency, supporting the validity of our screening strategy to isolate factors that alter cell wall properties related to saccharification. It is possible that these factors can alter SCW properties to increase enzymatic saccharification efficiency, e.g. the increased accessibility of enzyme. Or, they primary can inhibit the formation of SCW, which basically show negative effects for enzymatic saccharification, resulting in the increase of glucose yields.

In order to check effects of these factors on SCW formation, we next examined the growth and saccharification efficiency of inflorescence stems in 20 overexpressors that had high glucose yields in seedling samples. Growth inhibition was only observed in plants overexpressing VNI2, encoding a transcription factor that negatively regulates xylem formation (Yamaguchi et al. 2010; Supplementary Figure S2). The inflorescence stems were sampled from $10 \mathrm{~T}_{2} 40$-day-old overexpressors, and then the $10-\mathrm{cm}$ length of regions from the bottom were collected and subjected to the test of saccharification efficiency according to OkuboKurihara et al. (2016). In an enzymatic saccharification assay, eight and three lines showed significant increases and decreases in glucose yields from inflorescence

Table 1. Identification of genes that increase enzymatic saccharification efficiency.

\begin{tabular}{|c|c|c|c|c|c|c|}
\hline Gene ID & $\begin{array}{l}\text { Overexpressed } \\
\text { sequence }^{\mathrm{a}}\end{array}$ & Gene Symbols & Function Category $^{\mathrm{b}}$ & $\begin{array}{c}\text { Change in } \\
\text { glucose yeilds } \\
\text { (seedlings) }\end{array}$ & $\begin{array}{c}\text { Change in } \\
\text { glucose yeilds }{ }^{\mathrm{c}} \\
\text { (stems) }\end{array}$ & $\begin{array}{l}\text { Change in } \\
\text { xylose yeilds } \\
\text { (stems) }\end{array}$ \\
\hline AT1G64620 & pda12524 & DOF1.8 & TF (Dof type) & 160.47 & 106.23 & 113.37 \\
\hline AT5G15830 & pda10857 & BASIC LEUCINE-ZIPPER 3 (BZIP3) & TF (bZIP type) & 128.41 & 92.29 & 92.39 \\
\hline AT5G20710 & pda00366 & BETA-GALACTOSIDASE 7 (BGAL7) & CAZyme (GH35) & 125.00 & 103.70 & 98.38 \\
\hline AT1G22640 & pda03176 & MYB3 & TF (MYB type) & 123.66 & 92.32 & 106.57 \\
\hline AT1G02640 & pda01641 & BETA-XYLOSIDASE 2 (BXL2) & CAZyme (GH3) & 123.52 & 131.78 & 117.09 \\
\hline AT2G26730 & pda04099 & & LRR kinase & 119.30 & 119.77 & 97.97 \\
\hline AT3G42950 & pda04403 & & CAZyme (GH28) & 117.80 & 105.98 & 102.51 \\
\hline AT5G44030 & pda 13285 & $\begin{array}{l}\text { CELLULOSE SYNTHASE } 4 \text { (CESA4)/ } \\
\text { IRREGULAR XYLEM 5(IRX5) }\end{array}$ & CAZyme (GT2) & 113.87 & 85.43 & 106.24 \\
\hline AT3G16520 & pda00717 & UDP-GLUCOSYL TRANSFERASE 88A1 & CAZyme (GT1) & 113.73 & 110.31 & 104.94 \\
\hline AT3G18080 & pda02043 & B-S GLUCOSIDASE 44 & CAZyme (GH1) & 113.37 & 107.77 & 96.06 \\
\hline AT3G62720 & pda05997 & XYLOSYLTRANSFERASE 1 & CAZyme (GT34) & 112.53 & 119.09 & 101.23 \\
\hline AT2G28110 & pda08587 & $\begin{array}{l}\text { FRAGILE FIBER } 8 \text { (FRA8)/IRREGULAR } \\
\text { XYLEM } 7 \text { (IRX7) }\end{array}$ & CAZyme (GT47) & 111.84 & 106.74 & 95.99 \\
\hline AT5G20240 & pda11065 & PISTILLATA (PI) & TF (MADS-box type) & 111.80 & 88.23 & 89.87 \\
\hline AT3G15350 & pda03008 & & CAZyme (GT14) & 110.49 & 115.65 & 114.03 \\
\hline AT3G18660 & pda04027 & $\begin{array}{l}\text { GLUCURONIC ACID SUBSTITUTION } \\
\text { OF XYLAN 1(GUX1) }\end{array}$ & CAZyme (GT8) & 109.51 & 95.80 & 104.55 \\
\hline AT5G13180 & pda00727 & VND-INTERACTING 2 (VNI2) & TF (NAC type) & 108.59 & 107.89 & 109.62 \\
\hline AT4G24060 & pda00786 & DOF4.6 & TF (Dof type) & 107.98 & 113.85 & 120.99 \\
\hline AT2G30590 & pda09817 & WRKY21 & TF (WRKY type) & 107.96 & 103.95 & 101.85 \\
\hline AT1G19940 & pda04559 & GLYCOSYL HYDROLASE 9B5 & CAZyme (GH9) & 107.70 & 113.76 & 97.21 \\
\hline AT3G60140 & pda01565 & BETA GLUCOSIDASE 30 (BGLU30) & CAZYme (GH1) & 107.62 & 98.58 & 97.85 \\
\hline
\end{tabular}

${ }^{a}$ Arabidopsis full-length cDNA clones developed by RIKEN Genomic Sciences Center were used to generate overexpressors. ${ }^{\mathrm{b}} \mathrm{TF}$, transcription factor; CAZyme, carbohydrate active enzyme; GH, glycoside hydrolase; GT, glycosyltransferase. ${ }^{`}$ Three independent lines of overexpressors and control plants were subjected to enzymatic saccharification analysis, and the average yields of 3 lines were compared between overexpressors and control plants. Ratio of sugar yeilds of overexpressors to those of control plants were shown. Bold, statistically-significant different from the control (Student's $t$-test, $p<0.05$ ). 

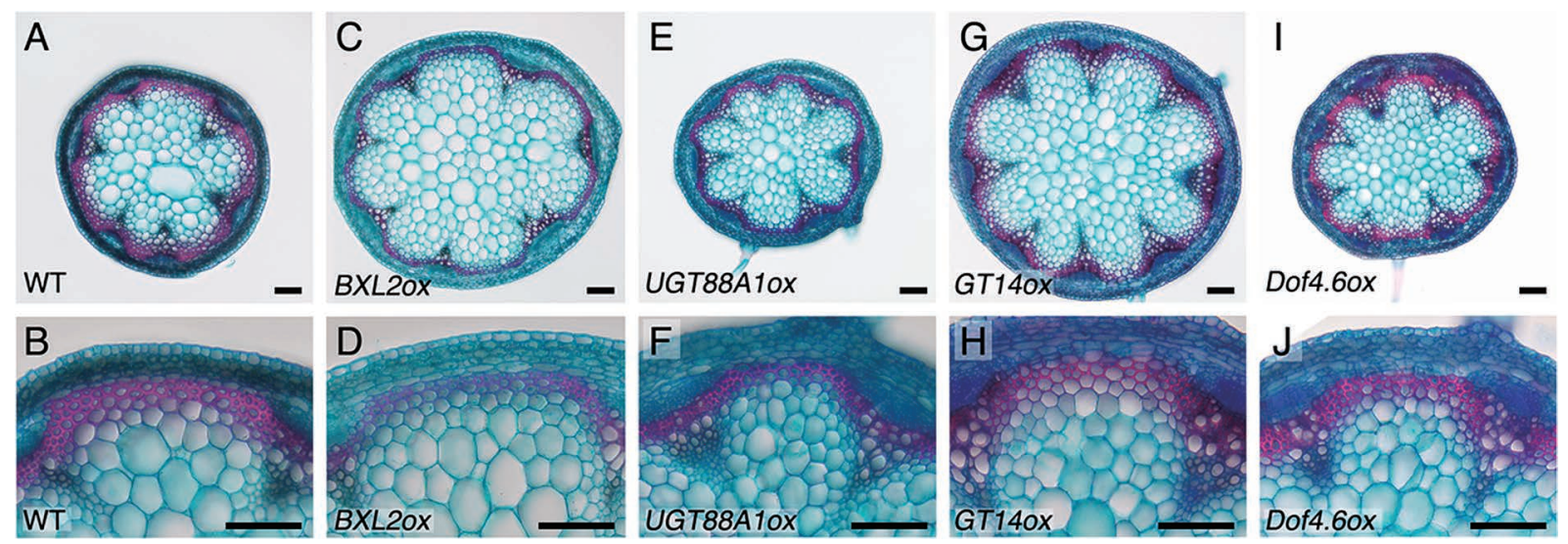

Figure 1. Transverse sections of mature inflorescence stems. The bottom regions of inflorescence stems were sampled from 40-day-old wildtype plants (A and B) and transgenic plants overexpressing BXL2 (BXL2ox; C and D), UGT88A1 (UGT88A1ox; E and F), AT3G15350 (putatively encoding a class GT14 glycosyltransferase; GT14ox; G and H), and Dof4.6 (Dof4.6ox; I and J) and fixed in fixative solution (90\% [v/v] ethanol, 10\% $[\mathrm{v} / \mathrm{v}]$ acetic acid) at $4^{\circ} \mathrm{C}$ overnight. Sections were prepared by hand and stained with safranine (red signals for lignin) and astra blue (blue signals for polysaccharides in cell walls). Bars, $100 \mu \mathrm{m}$.

stem samples, respectively (Table 1). These results suggest that the effects of overexpressing these genes on saccharification efficiency can differ between seedlings and inflorescence stems. The xylose yields, as determined using a D-Xylose Assay Kit (Megazyme), were also altered in five overexpressor lines, including lines overexpressing CELLULOSE SYNTHASE 4 (CESA4)/IRREGULAR XYLEM 5 (IRX5), BETA-XYLOSIDASE 2 (BXL2), UDP-GLUCOSYL TRANSFERASE 88A1 (UGT88A1), AT3G15350 (putatively encoding a class GT14 glycosyltransferase), and DOF4.6 (encoding a Doftype transcription factor) (Table 1). Among these, only the overexpression of CESA4/IRX5 had opposite effects on glucose vs. xylose yields, i.e., reduced glucose yields and increased xylose yields (Table 1). CESA4/IRX5 is an SCW-specific subunit of the cellulose synthase complex in Arabidopsis, which also contains the subunits CESA7/IXR3 and CESA8/IRX1 (Taylor et al. 1999, 2000, 2003). These three CESA proteins interact to form a functional cellulose synthase complex (Hill et al. 2014; Taylor et al. 2003). It is thus possible that overexpressing a single CESA gene has negative effects on cellulose synthase complex activity by disturbing the interactions among the three CESA proteins in inflorescence stems. The molecular functions of the four remaining genes described above in SCW formation have not yet been examined in detail, indicating that these four genes encode novel factors that potentially alter SCW properties to increase saccharification efficiency in wood tissues.

Finally, we examined the effects of these four genes on inflorescence stem structure by observing transverse sections of this tissue. We sectioned mature regions of inflorescence stems by hand and subjected them to double staining with safranin (red signals for lignin) and astra blue (blue signals for polysaccharides in cell walls) (Figure 1). Although the stem diameters tended to be larger in the BXL2 and AT3G15350 (GT14) overexpressors compared with the wild-type plants, no apparent defect in inflorescence stem structure, including vasculature and interfascicular fiber differentiation, was detected in any of the four overexpressors (Figure 1). Thus, in the case of the BXL2 and AT3G15350 (GT14) overexpressors, the ratio between cells with and without SCW within biomass samples could be changed, resulting in change of saccharification efficiency. In the BXL2 overexpressor, red signals from lignified cells appeared to be reduced in interfascicular fibers (Figure 1D), suggesting that the overexpression of BXL2 might affect lignification processes in wood tissues. The intensity and distribution of lignin signals were almost identical in the three remaining overexpressors compared to control plants (Figure 1). This result, together with the finding that these four overexpression lines did not show growth defects (Table 1; Supplementary Figure S3), suggests that these four genes might encode novel factors that can be used to increase saccharification efficiency in wood tissues without having negative effects on total biomass production.

In conclusion, we successfully isolated novel genes that alter saccharification efficiency in cultured Arabidopsis wood tissues by performing overexpression analysis of xylem vessel cell differentiation-related genes. The genes identified here can be used in molecular breeding strategies aimed at improving the utilization of lignocellulosic biomass of readily-decomposable woody plants. Moreover, the genes isolated in this study are expressed during xylem vessel cell differentiation (Kubo et al. 2005), suggesting their involvement in the process of woody cell differentiation. Further detailed analysis of these genes would enhance our understanding of the regulation of SCW biosynthesis in woody cells. 


\section{Acknowledgements}

We thank RIKEN Genomic Sciences Center for providing the fulllength Arabidopsis cDNA clones; RIKEN Bioresource Center for providing the plasmids for the overexpression FOX hunting system; Dr. Minami Matsui (RIKEN Center for Sustainable Resource Science) for technical support in Arabidopsis transformation; and Dr. Minoru Kubo, Dr. Ko Kato, Dr. Ryosuke Sano, Ms. Shizuka Nishida, and Ms. Eriko Tanaka (Nara Institute of Science and Technology, Japan) for critical discussions and technical support. This work was supported in part by RIKEN Center for Sustainable Resource Science, RIKEN Biomass Engineering Program, RIKEN Plant Science Center, the Japan Society for the Promotion of Science (KAKENHI Grant Number 25291062 to T.D.), and the Ministry of Education, Culture, Sports, Science, and Technology of Japan (Grant-in-Aid for Scientific Research on Innovative Areas "The Plant Cell Wall as Information Processing System" Grant Number 25114520 and 15H01235 to M.O., 24114002 to T.D., and Grants-in-Aid from the NC-CARP project to T.D.).

\section{References}

Clough SJ, Bent AF (1998) Floral dip: A simplified method for Agrobacterium-mediated transformation of Arabidopsis thaliana. Plant J 16: 735-743

Hill JL Jr, Hammudi MB, Tien M (2014) The Arabidopsis cellulose synthase complex: A proposed hexamer of CESA trimers in an equimolar stoichiometry. Plant Cell 26: 4834-4842

Hisano H, Nandakumar R, Wang ZY (2009) Genetic modification of lignin biosynthesis for improved biofuel production. In Vitro Cell Dev Biol Plant 45: 306-313

Ichikawa T, Nakazawa M, Kawashima M, Iizumi $H$, Kuroda $H$, Kondou Y, Tsuhara Y, Suzuki K, Ishikawa A, Seki M, et al. (2006) The FOX hunting system: An alternative gain-of-function gene hunting technique. Plant J 48: 974-985

Isikgor FH, Becer CR (2015) Lignocellulosic biomass: A sustainable platform for the production of bio-based chemicals and polymers. Polym Chem 6: 4497-4559

Khare SK, Pandey A, Larroche C (2015) Current perspectives in enzymatic saccharification of lignocellulosic biomass. Biochem Eng J 102: 38-44

Kubo M, Udagawa M, Nishikubo N, Horiguchi G, Yamaguchi M, Ito J, Mimura T, Fukuda H, Demura T (2005) Transcription switches for protoxylem and metaxylem vessel formation. Genes Dev 19: 1855-1860

Loqué D, Scheller HV, Pauly M (2015) Engineering of plant cell walls for enhanced biofuel production. Curr Opin Plant Biol 25: $151-161$
McCann MC, Carpita NC (2008) Designing the deconstruction of plant cell walls. Curr Opin Plant Biol 11:314-320

Myburg AA, Lev-Yadun S, Sederoff RR (2013) Xylem structure and function. In: eLS. John Wiley \& Sons Ltd, Chichester

Numata K, Ohtani M, Yoshizumi T, Demura T, Kodama Y (2014) Local gene silencing in plants via synthetic dsRNA and carrier peptide. Plant Biotechnol J 12: 1027-1034

Ohtani M, Demura T, Sugiyama M (2013) Arabidopsis ROOT INITIATION DEFECTIVE 1, a DEAH-box RNA helicase involved in pre-mRNA splicing, is essential for plant development. Plant Cell 25: 2056-2069

Okubo-Kurihara E, Ohtani M, Kurihara Y, Kakegawa K, Kobayashi M, Nagata N, Komatsu T, Kikuchi J, Cutler S, Demura T, et al. (2016) Modification of plant cell wall structure accompanied by enhancement of saccharification efficiency using a chemical, lasalocid sodium. Sci Rep 6: 34602

Pauly M, Keegstra K (2010) Plant cell wall polymers as precursors for biofuels. Curr Opin Plant Biol 13: 304-311

Sakamoto S, Mitsuda N (2015) Reconstitution of a secondary cell wall in a secondary cell wall-deficient Arabidopsis mutant. Plant Cell Physiol 56: 299-310

Seki M, Carninci P, Nishiyama Y, Hayashizaki Y, Shinozaki K (1998) High-efficiency cloning of Arabidopsis full-length cDNA by biotinylated CAP trapper. Plant J 15: 707-720

Seki M, Narusaka M, Kamiya A, Ishida J, Satou M, Sakurai T, Nakajima M, Enju A, Akiyama K, Oono Y, et al. (2002) Functional annotation of a full-length Arabidopsis cDNA collection. Science 296: 141-145

Taylor NG, Howells RM, Huttly AK, Vickers K, Turner SR (2003) Interactions among three distinct CesA proteins essential for cellulose synthesis. Proc Natl Acad Sci USA 100: 1450-1455

Taylor NG, Laurie S, Turner SR (2000) Multiple cellulose synthase catalytic subunits are required for cellulose synthesis in Arabidopsis. Plant Cell 12: 2529-2540

Taylor NG, Scheible WR, Cutler S, Somerville CR, Turner SR (1999) The irregular xylem 3 locus of Arabidopsis encodes a cellulose synthase required for secondary cell wall synthesis. Plant Cell 11: 769-780

Van Acker R, Vanholme R, Storme V, Mortimer JC, Dupree P, Boerjan W (2013) Lignin biosynthesis perturbations affect secondary cell wall composition and saccharification yield in Arabidopsis thaliana. Biotechnol Biofuels 6: 46

Yamaguchi M, Ohtani M, Mitsuda N, Kubo M, Ohme-Takagi M, Fukuda H, Demura T (2010) VND-INTERACTING2, a NAC domain transcription factor, negatively regulates xylem vessel formation in Arabidopsis. Plant Cell 22: 1249-1263 